\title{
Influence of the displacement effect on compressed LFM signal parameters
}

\author{
A. Kawalec, Cz. Leśnik, W. Komorniczak, W. Czarnecki \\ \& J. Pietrasiński \\ Military University of Technology, Institute of Radioelectronics, \\ Electronics Department, Warsaw, Poland
}

\begin{abstract}
This paper deals with the digital signal processing in the application of a modern radar receiver. The influence of the signal discretization and its side effects on radar performance are considered. In particular, the paper presents the problem of the random displacement effect of LFM (linear frequency modulation) radar echo pulse beginning in accordance with the closest discrete time value. This effect has an impact on the characteristics of the pulse compression filter output signal. Its equivalence to the Doppler frequency shift has also been proven. The shift is responsible for additional, unwanted amplitude modulation of the signal samples on the output of the matched filter, as a result of the discretization process. The resulting losses of the signal level (LPG) can reach $4 \mathrm{~dB}$. The losses follow the function of the sampling frequency, whose relation has been developed. In addition, the displacement effect can significantly decrease the effectiveness of the MTI process.

In the paper it has been shown that the influence of the displacement effect and discretization process on the relative level of the side lobes is not significant. The losses of PSL (Peak Side-lobe Level) do not exceed a fraction of dB. Characteristics evaluation of the discretization process has been performed on the basis of the proposed discrete time generation algorithm. The testing results are shown, as well as a discussion and some practical conclusions.

Keywords: signal processing, linear frequency modulation, radar signal, ambiguity function, signal discretization.
\end{abstract}




\section{Introduction}

The time representation of the signal at the matched filter output can be drawn from Woodward's general definition of the radar ambiguity function:

$$
\chi\left(\tau, f_{D}\right)=\int_{-\infty}^{\infty} u(t) u *(t-\tau) e^{j 2 \pi f_{D} t} d t
$$

where:

$u(t) \quad$ - signal complex envelope,

$u *(t) \quad$ - complex conjugate of $u(t)$,

$f_{D} \quad$ - Doppler frequency shift,

$\tau$ - relative time shift (related to time of maximum output signal).

During further consideration the radar ambiguity function modulus $\left|\chi\left(\tau, f_{D}\right)\right|$ will be taken into account. The most popular type of radar signal is a pulse with inner linear frequency modulation (LFM). Its normalized time representation is as follows $[1,2]$ :

$$
s(t)=\frac{1}{\sqrt{T}} \operatorname{rect}\left(\frac{t}{T}\right) \cos \left(\omega_{0} t+\frac{\mu t^{2}}{2}\right),
$$

where:

$T \quad$ - pulse duration,

$\omega_{0} \quad$ - carrier pulsation,

$\mu \quad$ - frequency slope, $\mu= \pm \frac{2 \pi B}{T} \quad\left[\mathrm{rad} / \mathrm{s}^{2}\right]$,

$B$ - total frequency deviation,

$\operatorname{rect}\left(\frac{t}{T}\right)$ - uniform rectangular pulse:

$$
\operatorname{rect}\left(\frac{t}{T}\right)=\left\{\begin{array}{ll}
1 & \text { for }-\frac{T}{2}<t<\frac{T}{2} \\
0 & \text { for } \text { other } t
\end{array}\right. \text {. }
$$

The complex signal envelope of (2) is represented by:

$$
u(t)=\frac{1}{\sqrt{T}} \operatorname{rect}\left(\frac{t}{T}\right) e^{j \frac{\mu t^{2}}{2}} .
$$

From (1) and (3) the equation describing the modulus of the LFM signal ambiguity function can be drawn [2]: 


$$
\left|\chi\left(\tau, f_{D}\right)\right|=\left|\operatorname{rect}\left(\frac{\tau}{2 T}\right)\left(1-\frac{|\tau|}{T}\right) \operatorname{Sa}\left[\left(2 \pi f_{D}+\mu \tau\right)\left(1-\frac{|\tau|}{T}\right) \frac{T}{2}\right]\right|,
$$

where:

$$
\operatorname{Sa}(x)=\left\{\begin{array}{ccc}
\frac{\sin x}{x} & \text { for } & x \neq 0 \\
1 & \text { for } & x=0
\end{array}\right.
$$

An expression (4) shows that a frequency axis position of the signal maximum at the matched filter output depends on Doppler frequency shift along with a modulation described by the function: $(1-|\tau| / T)$.

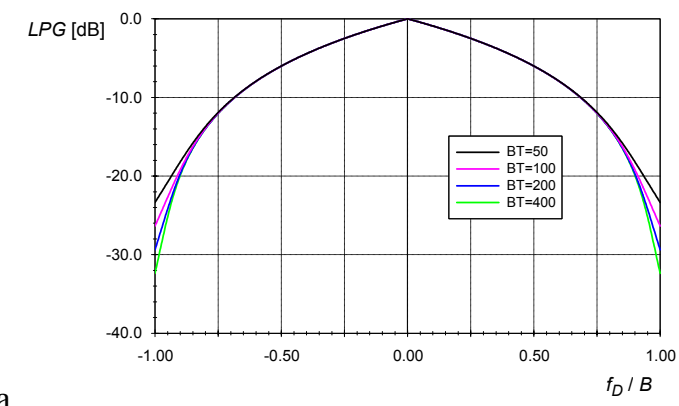

a.

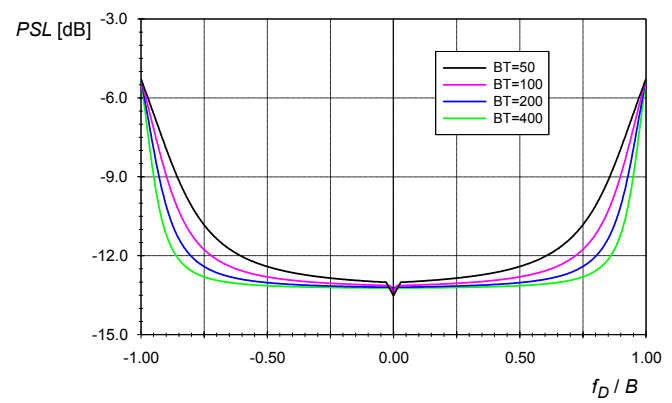

b.

Figure 1: $L P G$ (a.) and PSL (b.) values as a function of relative Doppler frequency shift.

Two of the most common matched filter output signal characteristics are as follows: LPG (Loss in Process Gain, the level of the mainlobe in no-match case $U_{M L}$ related to its maximum value in the perfect match case $U_{M L m x}$ ) and $P S L$ (Peak Sidelobe Level, relative level of the maximum sidelobe $U_{S L}$ related to the maximum of the mainlobe $U_{M L}$ ): 


$$
L P G=20 \log \frac{\left|U_{M L}\right|}{\left|U_{M L m x}\right|}, \quad P S L=20 \log \frac{\left|U_{S L}\right|}{\left|U_{M L}\right|}
$$

The $L P G$ and $P S L$ parameters as a function of the Doppler shift normalized to the deviation are derived according to equation (4). The characteristics for selected values of time-bandwidth product $B T$ are presented on Fig. 1 [4].

Minimum sidelobe level for zero Doppler shift is not a constant value, but it depends on time-bandwidth product BT. PSL parameter value fluctuations for low real (met in practice) values of Doppler frequency shift are presented on Fig. 2.

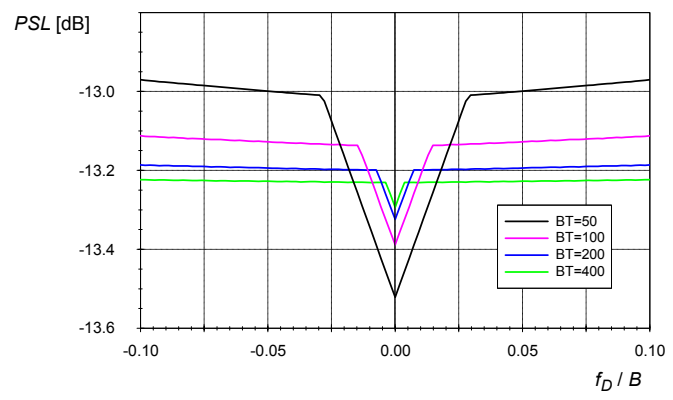

Figure 2: PSL values as a function of small values of relative Doppler frequency shift.

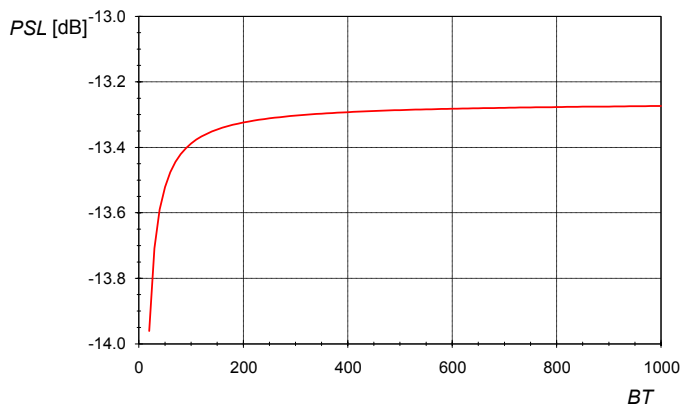

Figure 3: $\quad$ Minimum $P S L$ as a function of time-bandwidth product.

Sidelobe minimum level can be found as a result of looking for the second extreme of the function described by the expression (4) under assumption that $f_{D}=0$. Precise solution leads to very complicated expressions. That is why it is interesting to find the solution using numerical methods. Results of the calculations are shown on Fig. 3. 


\section{Radar signal discretization}

In the contemporary radar systems, the received echo signal is converted from its native analogue form to the digital representation. The conversion process is repeated with a sampling clock period $t_{s}$. The same period characterizes the set of samples which forms the response of the matched filter used for signal matched filtering. In the perfect condition the beginning of the echo signal overlaps with the sampling period beginning of the analogue-digital converter. In the general case, due to the random position of the detected object (relating to radar location), those two time moments do not overlap. The first signal sample is delayed from the beginning of the signal of the time $t_{j}$, which is shorter than $t_{s}$. The problem is illustrated on Fig. 4. The mentioned phenomenon is called a displacement effect.

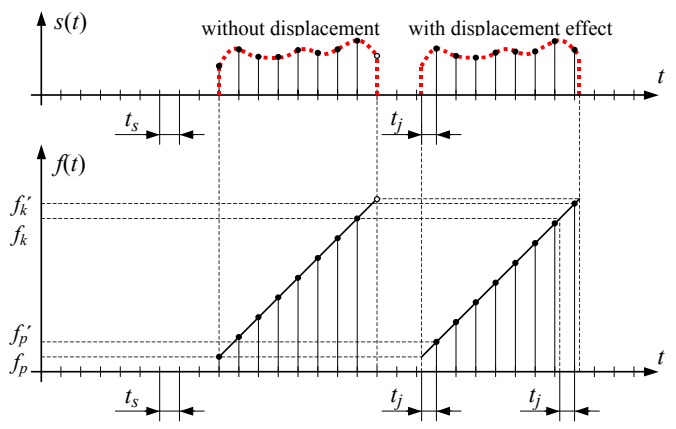

Figure 4: The displacement effect phenomenon.

The time shift fulfils the rule $t_{j} \in\left\langle 0, t_{s}\right)$. In the case of LFM signal, the temporary signal frequency is a linear function of time. In the idealized case the first and last sample of the received echo signal correspond to the values of initial $f_{p}$ and final $f_{k}$ frequency. In reality the first and last sample corresponds to the other initial $f_{p}^{\prime}$ and final $f_{k}^{\prime}$ frequency. As a result the frequency relations are going to be as on Fig. 5 .

The displacement effect results are equivalent to the Doppler frequency shift whose maximum value is given by:

$$
\Delta f_{m x}=\frac{\mu}{2 \pi} t_{s}
$$

According to equation (5), evaluation of the influence of the discretization process and displacement effect on the after compression signal characteristics can be reduced to examination of the Doppler frequency shift. 
The carrier frequency shift of the LFM signal causes changes of both the main signal lobe level at the matched filter output and the signal location on the time axis. It is shown on the Fig. 6. For simplification, presented signals are limited only to the first sidelobe pair.

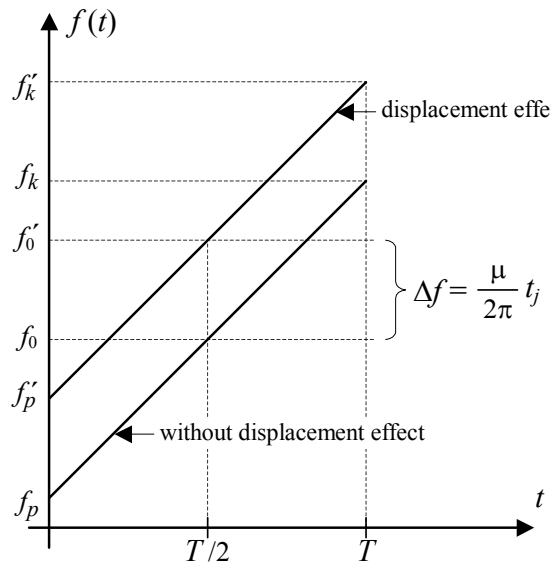

Figure 5: Temporary frequency in the cases of the lack and presence of the displacement effect.

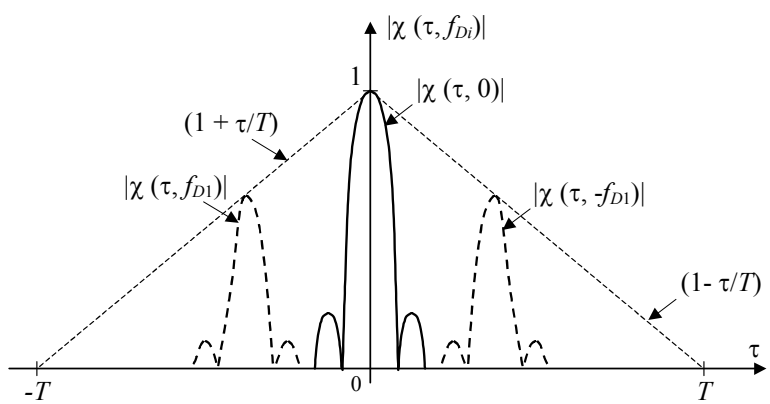

Figure 6: The output of the matched filter for chosen Doppler frequency shift values vs. time.

The time shift value can be found as an abscissa of the max of the function described by equation (4) for the constant $f_{D}$ value. Assuming $f_{d}=\Delta f$ and $t_{s}<<T$ the problem can be simplified to looking for the $\tau_{m x}$ value when the $\mathrm{Sa}[]$ function argument equals zero. Than

$$
\left(2 \pi \Delta f+\mu \tau_{m x}\right)\left(1-\frac{\left|\tau_{m x}\right|}{T}\right) \frac{T}{2}=\frac{\mu}{2}\left(t_{j}+\tau_{m x}\right)\left(T-\left|\tau_{m x}\right|\right)=0 .
$$


Finally one can conclude that the signal max time shift at the matched filter output caused by an effect mentioned above is described by an equation as follows

$$
\tau_{m x}=-t_{j}
$$

and in an extreme case it can reach its max value described as follows $\tau_{m x}=-t_{s}$. The minus sign means that the frequency shift caused by the discussed effect relates to a situation in which the Doppler frequency shift is an effect of a radial motion of a target towards a radar. As a result of the discretization process, the amplitude of the maximum mainlobe sample will deviate not only because of not being matched, but also in relation to the sampling clock (Fig. 7).

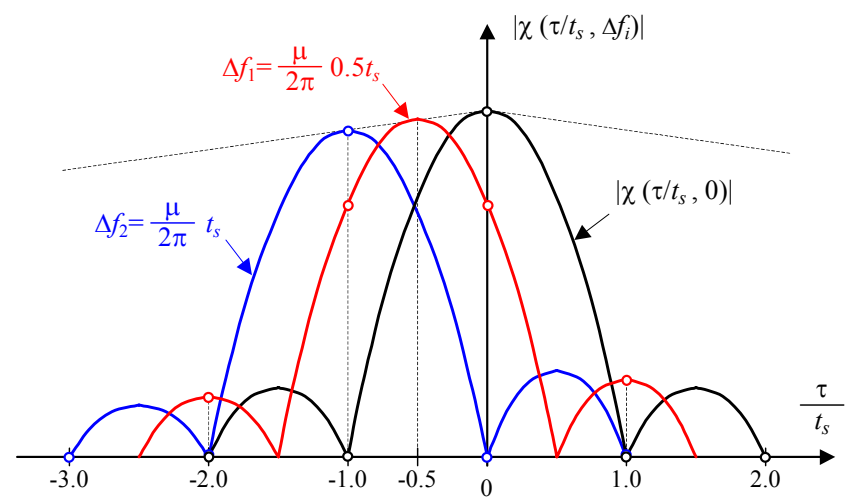

Figure 7: The influence of fluctuations on the samples amplitude after discretization.

The maximum loss concerning the mainlobe will be approximately $t_{j}=t_{s} / 2$. The amount of the loss is a function of the sampling period and it decreases with the sampling frequency increase. The maximum frequency, resulting from the Nyquist criterion, for the base band LFM signal is roughly equal to its frequency deviation $B$.

\section{Testing results}

The simulation model including the discretization process and displacement effect has been developed in order to evaluate the quantitative changes of the signal parameters on the output of the matched filter. As a result a series of characteristics have been obtained.

The parameter $L P G_{d}$ (for discrete time domain, for fixed time-bandwidth product $B T$ and several relative values of sampling frequency) as a function of $t_{j}$ is presented on Fig. 8. 
The value of $t_{j}$ is normalized to the maximum sampling period $t_{s m x}$ according to the Nyquist criterion. The figure also shows a characteristic of $L P G_{c}$ (continuous time domain) as a function of mean frequency of the signal in the time range corresponding to $t_{j}$. The losses of the output signal can reach as much as $4 \mathrm{~dB}$. In practice for sampling frequencies up to $2 B$ mentioned losses are still significant (about $1 \mathrm{~dB}$ ).

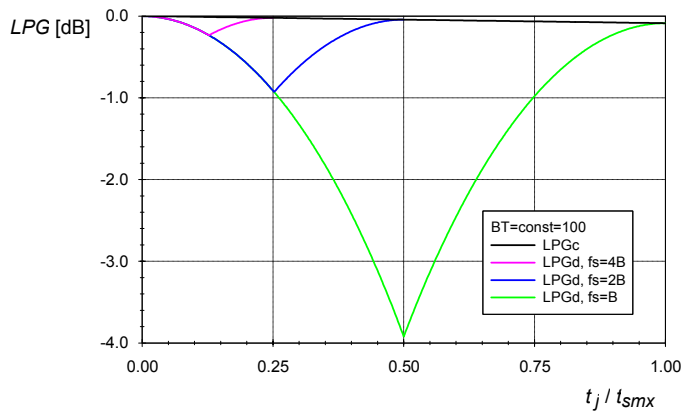

Figure 8: The relative level of the signal at the output of the matched filter as a function of $t_{j}$ and sampling frequency.

The lack of sensitivity of the parameter $L P G$ in relation to the signal base for several constant values of the sampling frequency is shown on Fig. 9. Presented characteristics demonstrate that there is no relation between the loss of the signal level as result of the displacement effect and the signal base. The changes in the level of maxima of the mainlobe $U_{M L}$ and sidelobes $U_{S L}$ after discretization are a result of the displacement effect at the matched filter output (Fig. 10). The presented characteristics are normalized to maximum values of the main and sidelobes for the continuous time domain and by no frequency shift.

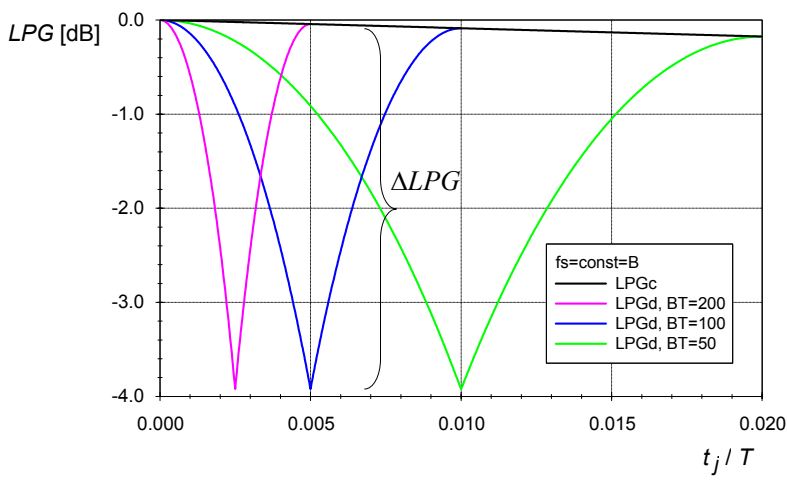

Figure 9: The relative level of the signal at the output of the matched filter as a function of $t j$ and the signal base. 


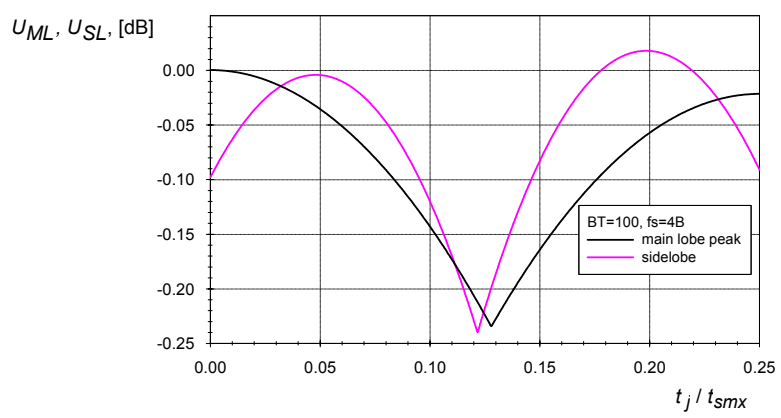

Figure 10: The maximum of the main and side lobes after discretization as a function of relative $t_{j}$.

Mentioned dependency results in the changes of the relative level of the sidelobes $P S L_{d}$. It is shown for two various values of the sampling frequency (Fig. 11). The figure also presents the $P S L_{c}$ parameter for continuous time domain in the range corresponding to the maximum value of $t_{j}$. Presented characteristics clearly show that the expected increment in the relative sidelobes level is not significant and does not exceed $0,1 \mathrm{~dB}$. Moreover, for some values of $t_{j}$ there is an improvement related to the level of the sidelobes, dependent on sampling frequency, and it exceeds $1 \mathrm{~dB}$.

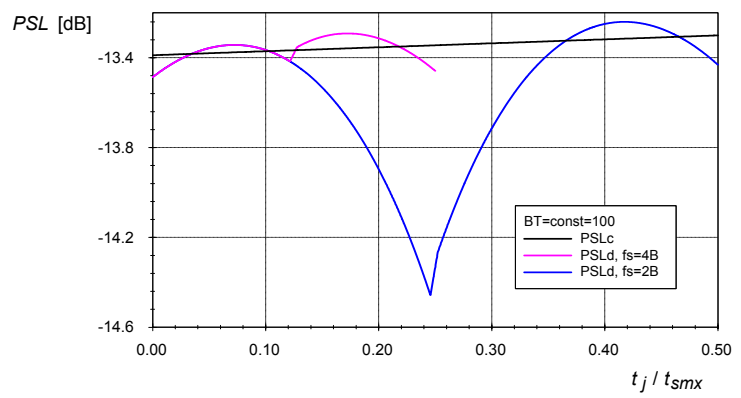

Figure 11: The relative level of the side lobes as a function of relative value of $t_{j}$ and the sampling frequency.

\section{Summary}

The paper presents the influence of discretization process on the LFM radar echo signal. The displacement effect between a pulse beginning and a closest time discrete value is examined. The equivalence of both displacement effect and Doppler frequency shift was proved. The frequency shift is responsible for additional, unwanted amplitude modulation of the signal samples at the matched 
filter output, as a result of the discretization process. The losses of the signal level $(L P G)$ can reach $4 \mathrm{~dB}$. The losses follow the function of the sampling frequency whose relation has been derived. In addition, the displacement effect can decrease the effectiveness of the MTI process.

In the paper it has been shown that the influence of the displacement effect and discretization process on the relative level of the sidelobes is not significant. The losses of PSL do not exceed a fraction of $\mathrm{dB}$.

\section{References}

[1] Cook C.E., Bernfeld M.: Radar Signals: An Introduction to Theory and Application. Artech House, Norwood, MA, 1993.

[2] Levanon N., Mozeson E.: Radar Signals. John Wiley \& Sons, Inc., Hoboken, New Jersey, USA, 2004.

[3] Peebles P.Z., Jr.: Radar Principles. John Wiley \& Sons, Inc., New York, USA, 1998.

[4] Kawalec A., Komorniczak W., Leśnik Cz., Pietrasiński J.: Discretization Process Impact on Compressed LFM Signal Parameters. Conference Proceedings, MIKON-2006, XVI International Conference on Microwaves, Radar and Wireless Communications, Poland, Kraków, May 22-26, 2006, vol. 3, pp. 1184-1187. 